\title{
Role of TEE in Improving Diagnostic Accuracy of Congenital Heart Disease
}

\author{
${ }^{1}$ Balbir Kumar, ${ }^{2}$ Sethu Madhavan J, ${ }^{3}$ Goverdhan Dutt Puri, ${ }^{4}$ Sandeep Singh Rana
}

\begin{abstract}
Complex congenital heart diseases (CHD) often present as multiple cardiac lesions. The presence of one anomaly should stimulate the physician to perform a comprehensive assessment and look for other associated anomalies. ${ }^{1}$ Multimodal imaging may be necessary to diagnose such associated lesions as single imaging may occasionally miss them. Transthoracic echocardiography (TTE) and transesophageal echocardiography (TEE) are complimentary to each other. Routine use of TEE during intraoperative period may help us to refine diagnosis, detect the missing components and guide effective surgical repair. We present one such case where diagnosis and management were optimized by multimodal imaging.
\end{abstract}

Keywords: Congenital heart diseases, Pediatric TEE, Shone complex.

How to cite this article: Kumar B, Madhavan JS, Puri GD, Rana SS. Role of TEE in Improving Diagnostic Accuracy of Congenital Heart Disease. J Perioper Echocardiogr 2014;2(2):68-70.

\section{Source of support: Nil}

Conflict of interest: None

\section{CASE SCENARIO}

Complex congenital heart diseases (CHD) usually present as a spectrum of diseases. ${ }^{1}$ A careful look for associated anomalies is necessary when suspected and multimodal imaging may be necessary to complete diagnosis. Lesions missed in one may turn up in another and a comprehensive imaging is advisable to formulate an optimal surgical plan. We discuss one such case where new lesions were picked up in intraoperative TEE and thus guided the surgeon.

\footnotetext{
1 Junior Resident, ${ }^{2}$ Senior Resident, ${ }^{3,4}$ Professor

${ }^{1-3}$ Department of Anesthesia and Intensive Care, Postgraduate Institute of Medical Education and Research Chandigarh, India

${ }^{4}$ Department of Cardiothoracic and Vascular Surgery, Postgraduate Institute of Medical Education and Research Chandigarh, India
}

Corresponding Author: Sethu Madhavan J, Senior Resident Department of Anesthesia and Intensive Care, Postgraduate Institute of Medical Education and Research, Chandigarh, India e-mail: smadhavan1983@gmail.com
A 6-year-old male child presented to our hospital with symptoms of dyspnea on exertion, failure to thrive and recurrent chest infections. Physical examination was normal except for a continuous murmur at left parasternal area. The physical and biochemical investigations were normal. The echocardiogram showed a large perimembranous ventricular septal defect (VSD) and single parachute mitral valve (Video 1). There was no coarctation of aorta or patent ductus arteriosus (PDA). The child was taken up for VSD closure and splitting of parachute mitral valve. After routine induction, invasive arterial lines were placed in the right radial artery and right femoral artery, which revealed a nonsignificant peak gradient of $15 \mathrm{~mm} \mathrm{Hg}$. A transesophageal echocardiography (TEE) probe (S7-3t pediatric TEE probe and iE33 xMatrix Ultrasound Systems, Philips, Bothell, WA, USA) was placed. TEE examination revealed single papillary muscle (Video 2) and an additional supramitral ring with flow acceleration (Fig. 1, Video 3). After heparinization, cardiopulmonary bypass (CPB) was initiated and heart arrested with cold cardioplegia. The surgeon excised the supramitral ring, split the parachute mitral valve and closed the VSD. As the gradient between radial and femoral was insignificant, no surgical intervention of aorta was done. The surgeon also informed of the presence of a subaortic membrane, which on retrospective review could be seen as a small bit of tissue in the TTE (Video 4). There was no significant

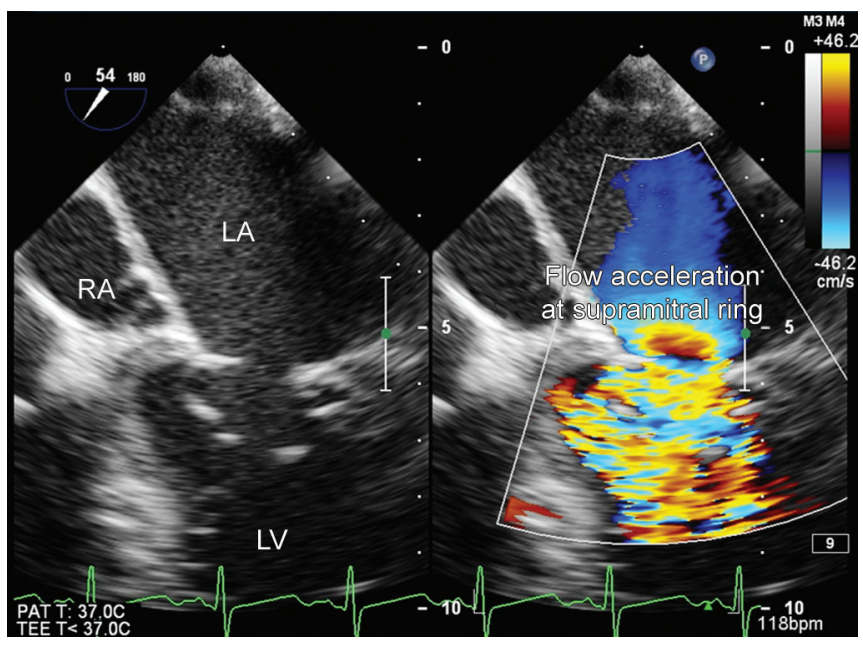

Fig. 1: Flow acceleration well above the level of mitral valve in mid esophageal commissural view (LA: Left atrium; RA: Right atrium; LV: Left ventricle) 


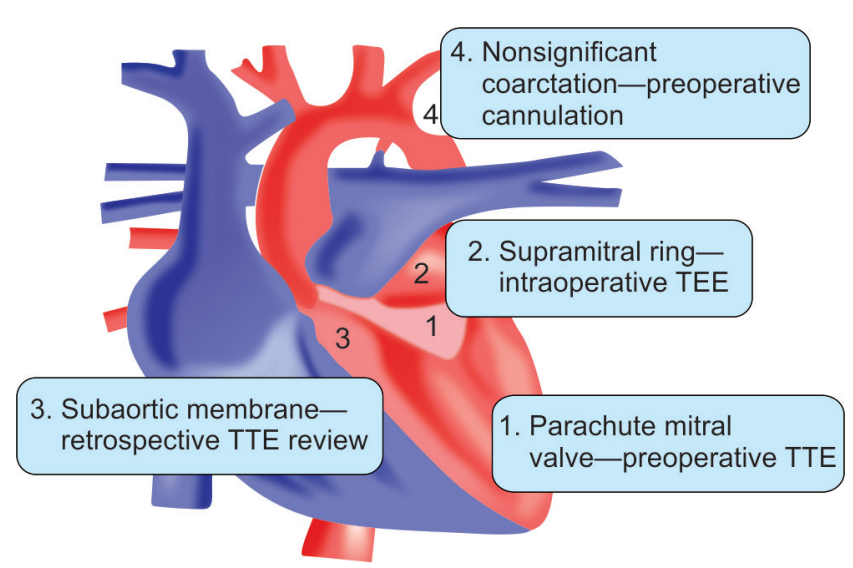

Fig. 2: The four components of Shone's complex and the role of transthoracic and transesophageal in the diagnosis (TTE: Transthoracic echocardiography; TEE: Transesophageal echocardiography)

gradient at LVOT both pre and post CPB. With all four components made out, parachute mitral valve (found on TTE preoperatively), gradient in the aorta (made by cannulating the radial and femoral arteries), supramitral ring (preoperative TEE) and subaortic obstruction (diagnosed retrospectively on review of echo), a diagnosis of Shone's complex could be made (Fig. 2). The child was weaned off $\mathrm{CPB}$ on milrinone and adrenaline as per institute protocol and had an uneventful postoperative course. A written informed consent was obtained from the parents for reporting the case and publishing the echocardiography images and videos.

\section{DISCUSSION}

Shone's, complex is a syndrome consisting of four components, first described by Shone et al. ${ }^{2}$ Absence of any one of these component forms an incomplete Shone's complex, which is more common than the complete

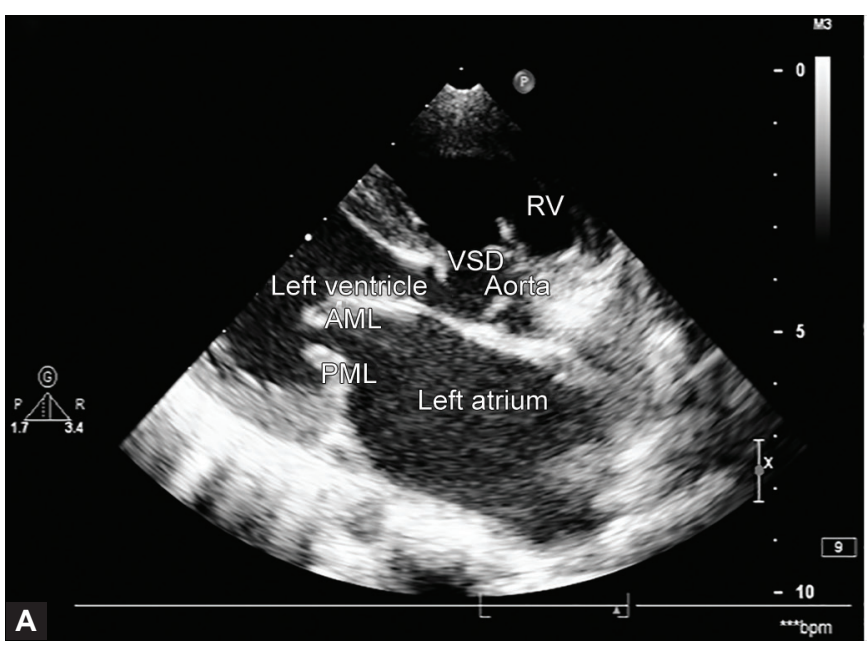

Figs $3 \mathrm{~A}$ and $\mathrm{B}$ : Equivalent views of transthoracic and transesophageal echocardiography. While the supramitral ring cannot be appreciated in parasternal long-axis view in transthoracic echo (A), the same can be visualized (white arrow) in mid esophageal aortic valve long-axis view in transesophageal echo (B) due to proximity to left atrium (RV: Right ventricle; AML: Anterior mitral leaflet; PML: Posterior mitral leaflet; LA: Left atrium; LV: Left ventricle; VSD: Ventricular septal defect)

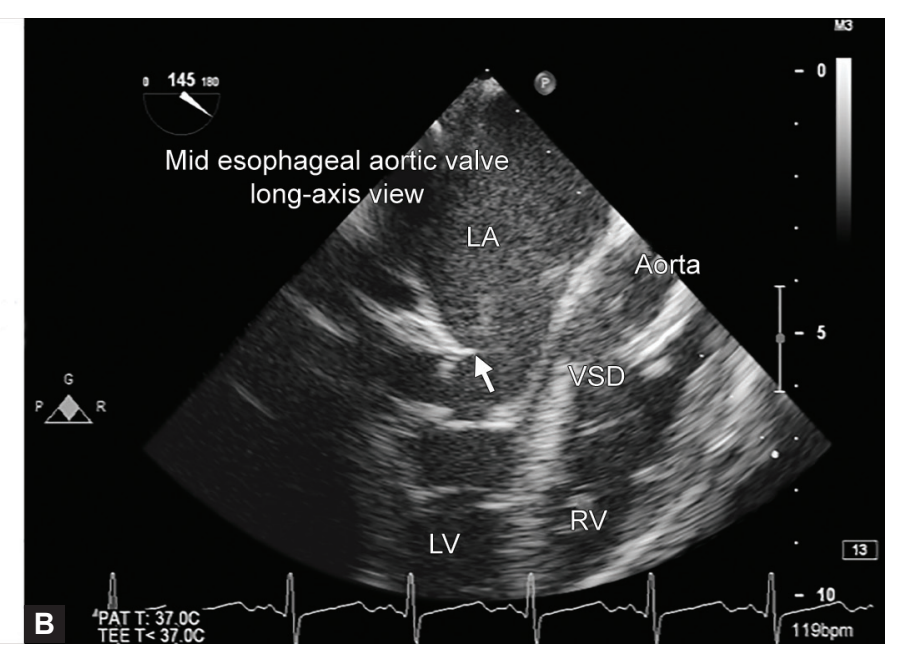

form. ${ }^{1}$ Suspected to be due to mitral inflow obstruction during fetal development, it leads to under development of left ventricle (LV) and varying degrees of left outflow obstruction. ${ }^{1}$ Diagnosis of Shone's complex has been made in varying age groups. ${ }^{3}$ The diagnosis of these components can be made on TTE, but TEE has been shown to have an incremental value in the diagnosis of parachute mitral valve, ${ }^{4}$ especially in older children and adults. TEE is also useful in demonstrating other associated lesions like atrial septal defects and atrial thrombi ${ }^{5}$ in patients taken up for repair.

Use of TEE has been strongly recommended by American Society of Anesthesiology/Society of Cardiovascular Anesthesiologists (ASA/SCA) guidelines in all open cardiac procedures. ${ }^{6}$ The proximity of TEE to the left atrium (LA), mitral valve (MV) and the absence of interference from lung tissue make it an ideal tool for close evaluation of LA and MV (Figs 3A and B). Routine use of TEE during intraoperative period has been found to be cost effective and reduces the need for reoperations. ${ }^{7}$ TEE has advantage over epicardial imaging as sterility is not compromised and there is no need to interrupt surgery for imaging. ${ }^{7}$ Cases, such as Shone's complex, which have been associated with high mortality may benefit from TEE to ensure adequate surgical correction. Mitral valve repair is considered superior to replacement and, hence, TEE plays a vital role in guiding the adequacy of correction.

Use of TEE probe is not without complications. Incidences of difficulty in ventilation due to high airway pressure and accidental dislodgement of endotracheal tube have been described. ${ }^{6}$ Despite these complications, the risk benefit ratio needs to be assessed for individual cases and TEE should be used in every possible case. 


\section{REFERENCES}

1. Popescu BA, Jurcut R, Serban M, Parascan L, Ginghina C. Shone's syndrome diagnosed with echocardiography and confirmed at pathology. Eur J Echocardiogr 2008;9:865-867.

2. Shone JD, Sellers RD, Anderson RC, Adams P, Lillehei CW, Edwards JE. The developmental complex of 'parachute mitral valve,' supravalvular ring of left atrium, subaortic stenosis and coarctation of aorta'. Am J Cardiology 1963;11:714-725.

3. Hakim FA, Kendall CB, Alharthi M, Mancina JC, Tajik JA, Mookadam F. Parachute mitral valve in adults-a systematic overview. Echocardiography 2010;27:581-586.

4. Shapira OM, Connelly GP, Shemin RJ. Ischemic papillary muscle dysfunction in an adult with a parachute mitral valve. J Cardiovasc Surg (Torino) 1995;36:163-165.
5. Prunier F, Furber AP, Laporte J, et al. Discovery of a parachute mitral valve complex (Shone's anomaly) in an adult. Echocardiography 2001;18:179-182.

6. Practice guidelines for perioperative transesophageal echocardiography: an updated report by the American Society of Anesthesiologists and the Society of Cardiovascular Anesthesiologists Task Force on Transesophageal Echocardiography. Anesthesiology 2010;112: $1-13$.

7. Misra A, Madhavan JS, Ghuman BPS, Raj R, Kumar A, Dutta $\mathrm{V}$, et al. Impact and cost effectiveness of routine intraoperative transthoracic and transesophageal echocardiography on surgical decision making in pediatric cardiac surgery. J Perioper Echocardiogr 2014;2:3-9. 